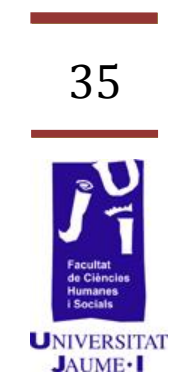

\title{
La Represión de los Crímenes Internacionales por parte de los Tribunales Nacionales: el Alien Tort Claims Act
}

Maria Chiara Marullo mariachiara.marullo2@unibo.it 


\section{Resumen}

El verdadero desafío de la Comunidad Internacional es determinar las fuerzas, los poderes y las normas que regulan a la sociedad internacional en un determinado momento histórico. Éste se concretiza en el establecimiento de los límites y criterios útiles a la hora de determinar la primacía, la subordinación o la compatibilidad entre las normas internacionales o entre estas últimas y las normas nacionales.

En los últimos años, por ejemplo, el debate internacional se ha centrado sobre la temática relativa a la jurisdicción universal en materia civil en caso en que se haya concretizado una violación de una norma de derecho internacional que prohíbe la comisión de un crimen internacional. La jurisdicción civil universal es una norma que otorga competencia a los tribunales nacionales para indemnizar a las victimas de crímenes internacionales.

Otorgando competencia civil de carácter universal sobre crímenes que han sido cometidos en el extranjero por parte de extranjeros, tropieza con otras leyes internas e internacionales y debido a esto, ha encontrado numerosos obstáculos para su aplicabilidad. Para complicar aún más la situación, hay que tener en cuenta otros aspectos como el hecho de ser actualmente adoptada por un solo país, Estados Unidos de América, a través del Alien Tort Claims Act.

Partiendo del análisis histórico sobre la utilización de esta tipología de jurisdicción, el presente artículo se centrará en el corazón de la temática relativa a los límites y críticas que rodean esta norma hacia la creación de una responsabilidad sobre las empresas en la comisión de crímenes internacionales. Precisamente la incertidumbre que rodea esta ley desde el momento de su creación hace mas difícil determinar y solucionar los diferentes problemas a los que los tribunales nacionales se enfrentan en cada caso basado en esta jurisdicción.

Palabras claves: Jurisdicción Civil Universal - Crímenes Internacionales Tortura - Alien Tort Claims Act - Indemnización.

\section{Introducción}

Cuando se habla de la competencia para perseguir un crimen, en general se reconocen cinco principios básicos para su atribución a los tribunales nacionales: el principio de territorialidad, el principio de nacionalidad o personalidad activa, el principio de nacionalidad o personalidad pasiva, el principio de protección de intereses nacionales, y el principio de jurisdicción universal.

El principio de territorialidad tiene prioridad en la medida en que el lugar de comisión del crimen, locus delicti, es la base principal para el ejercicio de la jurisdicción estatal. En base a este criterio es competente a juzgar un delito el juez del lugar donde el mismo ha sido cometido. Como 
explica Beccaria, en su libro sobre los delitos y las penas, la territorialidad es otra forma del concepto mismo de soberanía «il luogo della pena è il luogo del delitto, perché ivi solamente, e non altrove, gli uomini sono sforzati di offendere un privato per prevenire l'offesa pubblica» ${ }^{1}$ (Beccaria, 1764: 47).

Podemos decir que este último pensamiento representa el fundamento ideológico de la territorialidad en cuanto es en el lugar en que se comete un crimen donde debería actuarse su represión. Por otro lado, podemos encontrar un fundamento más práctico del concepto de territorialidad en cuanto es en el lugar en que se cometió un delito donde, en la normalidad de las circunstancias, se encuentran las pruebas, los testigos, el reo etc. "En el sistema tradicional, cada Estado persigue los crímenes cometidos en su territorio. En base a su soberanía, se le impone la responsabilidad de asegurar el orden público en su territorio» (Tomuschat, 2009:11).

Este principio bien se adapta para la represión de lo que se denominan crímenes comunes, como un asesinato, un hurto, una agresión, delitos que por su naturaleza se desarrollan en un único momento, o en un mismo plan criminal y en un único espacio en que sucesivamente se descubren las pruebas, los testigos etc. Pero cuando se trata de crímenes que por su propia naturaleza implican la responsabilidad del gobierno de un Estado, o que se cumplen bajo el control o el apoyo de aparatos gubernamentales, o delitos que implican la responsabilidad de un gran número de sujetos y que afectan a poblaciones enteras y que trascienden un único Estado, este criterio encuentra muchos obstáculos que traen como consecuencia primaria la impunidad más amplia para las personas implicadas en estos actos. Por esta razón, el criterio de territorialidad junto con el de la pasividad de las víctimas, poco a poco ha sido sustituido, por criterio de universalidad, basado únicamente en la naturaleza del crimen.

Con ello se ha incrementado el debate respecto del papel de la jurisdicción universal en materia civil como mecanismo de reparación de violaciones graves a los Derechos Humanos. Esta jurisdicción ha sido utilizada como una de las alternativas posibles, a modo de respuesta útil para la represión y prevención de crímenes como la tortura o el genocidio. En base a esta norma se da la posibilidad de dar una respuesta reparadora a las víctimas que han sufrido uno de estos crímenes, en un Estado que no está ligado al crimen mismo, o que no tiene ningún interés específico en la represión y reparación del delito.

Desde ahora resulta importante decir que en tema de protección de los Derechos Humanos, la jurisdicción territorial tendrá una importancia peculiar, siempre y cuando se demuestre que sea realmente efectiva y eficaz. En este sentido podemos definir la jurisdicción universal como un mecanismo de carácter excepcional y que por lo tanto, a nivel nacional

\footnotetext{
${ }^{1}$ Traducción: el lugar de la pena es el lugar del delito, porque solamente allí, y no en otra parte, los hombres se han esforzado en ofender a un privado para prevenir una ofensa pública.
} 
como internacional debe ser regulado con claridad y ejercerse con el fin de proteger los valores fundamentales de la Comunidad Internacional previniendo de las violaciones a sus valores e intereses compartidos.

La idea puesta a fundamento de este principio es que, cuando se trata de crímenes tan graves que ofenden el concepto mismo de humanidad y que se quedarían en el olvido por parte del Estado en que se verificaron los actos, resulta necesario poner en marcha instrumentos útiles para la indemnización de los daños sufridos por las víctimas aunque estamos en una situación en que se procede por crímenes cometidos en el extranjero, por extranjeros en contra de otros extranjeros.

El único ejemplo de Jurisdicción Civil Universal está representado por la Ley estadounidense sobre la indemnización civil por daños extra contractuales a extranjeros, denominada Alien Tort Statute or Alien Tort Claims Act (desde este momento ATCA) de 1789; podemos ver como dicha ley otorga competencia a las cortes federales de distrito para conocer casos en que un extranjero reclame violaciones al derecho internacional o a los tratados internacionales en que Estados Unidos de América es parte. Este acto ha sido codificado en 2004 en la Constitución de los Estados Unidos de América en su artículo 28 párrafo 1350 que prevé: "The district courts shall have jurisdiction of any civil action by an alien for a tort only, committed in violation of the law of nations or a treaty of the United States $)^{2}$. Como explica el Profesor Beigbeder (2005: 57), esta ley otorga una competencia a las cortes federales limitadas a conocer la sola responsabilidad civil para la violación de los Derechos Humanos y a indemnizar las víctimas:

The US Courts' jurisdiction under this Act is limited to adjudicating civil liability for human rights violations (especially torture). The Courts have civil, not criminal, competence: they do no sanction the perpetrators except by deciding on restauration to the victms, limited to finacial compensation ${ }^{3}$.

Para la activación de este acto resultan necesarios tres elementos establecidos por la Corte del decimo primer circuito en la sentencia del 2009 sobre el caso relativo a Sinaltrainal v. Coca-Cola Co., (578 F.3d 1252 (11th Cir. 2009)): «Federal subject matter jurisdiction exists for an ATS claim when the following three elements are satisfied: (1) an alien (2) sues for a tort (3) committed in violation of the law of nations ${ }^{4}$. Sobre el primer punto, en la jurisprudencia constante de las cortes federales,

\footnotetext{
${ }^{2}$ Traducción: Los tribunales de distrito tendrán jurisdicción sobre cualquier acción civil activada por un extranjero solamente por un acto ilícito cometido en violación de la ley de las naciones o un tratado de los Estados Unidos.

${ }^{3}$ Traducción: Competencia de los tribunales de EE.UU. en virtud de esta Ley se limita a determinar la responsabilidad civil por violaciónes de derechos humanos (especialmente por tortura). Los tribunales tienen una competencia civil, no penal: no establecen ninguna sanción a los responsables, excepto por la determinacion de la restauración de las victimas, limitada a la compensación financiera.

${ }^{4}$ Traducción: La jurisdicción federal existe bajo el ATS cuando una demanda respete los tres siguientes elementos: (1) un extranjero (2) demanda por responsabilidad extracontractual (3) cometido en violación del derecho de gentes.
} 
podemos decir que este acto no está limitado a los extranjeros sino bien a los individuos, también ciudadanos estadounidenses, que resultan ser víctimas de tortura, genocidio u otro crimen en contra de la humanidad. Más adelante veremos en qué consisten los torts cometidos en violación de la law of nations, y la evolución jurisprudencial sobre estos aspectos.

Como confirmación de la importancia de una legislación que pudiese indemnizar las victimas de crímenes internacionales, el congreso en 1992 codificó otro importante acto: The Torture Victim Protection Act de 1992 (desde ahora TVPA). Este acto autoriza a cualquier individuo, nacional o extranjero, a reclamar civilmente la comisión de actos de tortura o ejecuciones extrajudiciales, siempre y cuando no hayan tenido un acuerdo de solución en el lugar de comisión de tales acciones. Este documento ha sido codificado en el artículo 28 párrafo 1350 de la Constitución de los Estados Unidos Americanos. La idea de fondo que sustenta esta norma es clara, con su creación: «lt highlights the role of U.S. Courts in providing a legal forum for outrageous violations of human rights regardless of where they are committed ${ }^{5}$ " (Koebele $2009: 5$ ).

Resulta importante decir que el ejercicio del principio de jurisdicción universal en materia civil se encuentra aún en formación en el ámbito internacional y que su futuro es incierto, esto por las dificultades que se encuentran para llevarlo a cabo. No existen normas de derecho internacional que obliguen a los Estados a ejercitar la jurisdicción civil universal en los casos de violaciones de las normas sobre los Derechos Humanos, aunque muchos tratados internacionales faculten a los Estados a ejercitar una jurisdicción penal universal en caso de genocidio, crímenes contra la humanidad, crímenes de guerra, crimen esclavitud y de piratería.

\section{La experiencia estadounidense a partir de la ley sobre indemnización civil por daños extra-contractuales causados a extranjeros}

El ejemplo más significativo, prácticamente el único, de ejercicio de esta jurisdicción civil está representado por la legislación de Estados Unidos de América. La legislación estadounidense ha jugado un papel muy destacado; aunque también ha sido objeto de críticas, tanto internas como externas, las cuales han llevado a una interpretación de dicha legislación de forma variable y, en los últimos tiempos, cada vez más restrictiva. Como sostiene Mauricio Iván Del Toro Huerta:

La experiencia estadounidense es ilustrativa respecto del papel de los tribunales nacionales frente a la protección de los Derechos Humanos y sobre la falta de mecanismos efectivos de reparación de las violaciones graves a tales derechos,

\footnotetext{
${ }^{5}$ Traducción: Se destaca el papel de los tribunales de los EE.UU. en ofrecer un foro legal para violaciones escandalosas de los derechos humanos, independientemente del lugar en que se cometió
} 
aspectos fundamentales en la construcción de una comunidad nacional e internacional más humana y más justa (Del Toro Huerta, 2007: 3).

Al finales de 1700, después de muchos accidentes sufridos por diplomáticos extranjeros en el territorio estadounidense, el Congreso decidió adoptar una ley que establecía una jurisdicción universal en materia civil que podría ser actuada frente a los tribunales nacionales estadounidenses para las violaciones de la law of nations o de los treaty of the United States.

La ley llamada Alien Tort Claims Act, sobre los daños extracontractuales causados a extranjeros, fue promulgada en 1789 , pero por casi dos siglos no fue empleada de forma significativa y no existieron sentencias relevantes de parte de las cortes estadounidenses. Antes de 1980 , solo un número menor de casos merecieron la aplicación de esta ley. La situación cambió con el caso Filartiga contra Peña-Irala (1980), en el que un ciudadano paraguayo demandó a un antiguo alto oficial de policía de la dictadura militar de Strossner en el Paraguay por actos de tortura cometidos en dicho Estado, actos que llevaron a la consecuencia de la muerte del hijo menor de edad del demandante. En este caso, la Corte de Apelación del Segundo Circuito, con efecto de establecer la competencia del tribunal con base en el derecho internacional general, equiparó en sus efectos el crimen de piratería con el de tortura al señalar que:

Among the rights universally proclaimed by all nations, as we have noted, is the rights to be free of physical torture. Indeed, for purposes of civil liability, the torturer has become like the pirate and slave trader before him hostis humani generis, an enemy of all mankind ${ }^{6}$. (Sentencia: Filártiga v. Peña-Irala, 630 F.2d 876 2d Cir.1980).

En consecuencia, y considerando la opinión favorable de la administración del ex presidente Carter, dicha corte se consideró competente y estimó que «deliberate torture perpetrated under color of official authority violates universally accepted norms of the international law of human rights ${ }^{7} »$. Esta sentencia representa un punto muy importante en la historia del ATCA pero sobre todo un momento significativo para la protección de los Derechos Humanos.

In terms of the international protection of human rights, this decision must count as one of the most significant judicial judgments ever rendered, placing the ATS at the center of human rights adjudication. This focus necessarily raised

\footnotetext{
${ }^{6}$ Traducción: Entre los derechos universalmente proclamados por todas las naciones, como hemos señalado, es el derecho a estar libre de tortura física. De hecho, para efectos de la responsabilidad civil, el torturador, es ahora un pirata y comerciante de esclavos, hostis humani generis, un enemigo de toda la humanidad.

7 Traducción: tortura deliberada perpetrada bajo la apariencia de autoridad oficial viola normas universalmente aceptadas del derecho internacional de los derechos humanos.
} 
questions about the scope and limits of claims under this statute ${ }^{8}$.(Caron $y$ Buxbaum;2010: 2).

Desde el caso Filartiga, se han presentado numerosas demandas por actos cometidos fuera del territorio Estadounidense, en perjuicio de extranjeros, inaugurándose una nueva era jurisprudencial de dicho acto. Después de este caso, la historia de la aplicación de esta norma ha conocido demandas por diferentes violaciones de normas internacionales que se concretizan en diversos crímenes internacionales, entre ellos podemos mencionar: tortura, genocidio, violencia sexual, crímenes de guerra, esclavitud y trabajos forzosos, apartheid, ejecuciones extrajudiciales, tratos inhumanos y degradantes, daños graves al ambiente y desapariciones forzadas.

A nivel histórico podemos identificar tres diferentes periodos en la Historia del ATCA: el primero es el periodo pre-Filartiga, es decir el periodo que va de 1789 al 1980, donde el estatuto no ha sido utilizado sino en casos marginales; el segundo periodo parte del caso Filartiga y llega hasta el 2004 con el Caso Sosa. Se abre una nueva etapa, el ATCA empieza a ser utilizado en casos de crímenes de tortura, de Genocidio o en contra de la Humanidad. Finalmente una tercera fase post caso Sosa, todavía no concluida, etapa en la cual la Suprema Corte confirma la importancia del Estatuto y abre el debate relativo a los criterios de aplicabilidad del ATCA, es referente a la responsabilidad de las empresas en la comisión de crímenes internacionales y sobre la compatibilidad de la norma que prevé la indemnización para los daños extra contractuales y las normas que otorgan inmunidad a los Estados extranjeros de la Jurisdicción civil y penal.

A lo largo de estas tres fases, los criterios, las características y los efectos de esta tipología de jurisdicción han sido diferentes y han llevado a numerosos fallos y críticas de este sistema. Los casos Filartiga y Sosa por diferentes aspectos, representan momentos de cambio significativos y determinan las líneas jurisprudenciales seguidas por las cortes federales en un determinado momento.

El caso Filartiga pone en evidencia el problema de adaptar una norma creada en 1789, por problemas y amenazas a la seguridad internacional y a la paz, muy diferentes de las que en 1980 las cortes tenían que sancionar. El tribunal tenía el arduo papel de determinar si los actos de torturas estaban o no cubiertos por el dictado del ATCA original. Como hemos mencionado antes, el congreso decidió otorgar competencia universal solamente en casos en que se había cometido una violación de la ley de las naciones. La expresión ley de las naciones, como bien explica el Profesor Tanzi (2010:114), es propia del derecho internacional consuetudinario y por lo tanto el Juez en este caso pudo

\footnotetext{
${ }^{8}$ Traducción: En cuanto a la protección internacional de los derechos humanos, esta decisión debe contar como una de las sentencias judiciales más importantes jamás emitidas, colocando el ATS en el centro de la adjudicación de derechos humanos. Este enfoque necesariamente plantea preguntas sobre el alcance y los límites de las reclamaciones en virtud de este estatuto
} 
declarar su competencia a conocer la causa solo determinando la naturaleza consuetudinaria de la norma internacional que prohíbe los actos de tortura.

Tenendo conto che l'espressione the law of nations, il c.d. diritto delle genti, corrisponde al diritto internazionale consuetudinario e poiché gli attori avevano basato l'azione in giudizio sulla consuetudine, per determinare la propria giurisdizione il giudice americano ha dovuto accertare la natura consuetudinaria del divieto della tortura ${ }^{9}$.

La decisión de la Suprema Corte en el caso Sosa v. Alvarez Machain (542 U.S. 692 (2004)), ciertamente representa un punto de ruptura con el pasado y con la líneas seguidas por los tribunales en el caso Filartiga y un punto de partida para entender la actual utilización del ATCA. De hecho dicha Corte, diferentemente de la jurisprudencia precedente, estableciò que el ATCA no establece un derecho o causa de acción propiamente dicha, sino que reconoce la jurisdicción de los tribunales federales sobre un cierto número limitado de crímenes de derecho internacional. Podemos ver que en este caso, contrariamente a la sentencia Filartiga, se opto por un criterio restringido en el determinar cuáles pueden ser los crimines punibles con el ATCA, es decir: las violaciones que teniendo un elevado grado de especificidad, universalidad y obligatoriedad podrían ser incluidas en el listado de violaciones recogida en dicha norma, siguiendo las interpretaciones hechas originalmente por el Congreso en el momento de su creación.

Este cambio ha sido posible en cuanto la interpretación del ATCA esta en las manos de las cortes federales que determinan, a través de sus sentencias, también los criterios aplicables para establecer, por ejemplo, cuales crímines entran o no entran en el dictado normativo.

En general la sentencia en el caso Sosa vs. Alvarez-Machain representó un parte aguas en la dinámica que hasta el momento había tenido la aplicación del ATCA. En un intento por equilibrar los diferentes intereses en juego, en su fallo salomónico, la corte, por un lado, reconoció que en principio la ley no establece propiamente un derecho de acción abierto sino sólo una competencia jurisdiccional, por el otro, reconoció también la posibilidad de que un número muy limitado de violaciones al derecho internacional puedan ser alegadas en el marco del ATCA (Del Toro Huerta, 2007).

El efecto determinado de esta sentencia ha tenido seguramente un alcance elevado y ha enriquecido los debates y las críticas sobre un sistema de justicia universal que ha tenido, tiene y tendrá numerosos defectos debido a causas estructurales y ligadas a la creación de una norma que durante siglos no ha sido modificada y adaptada a las nuevas exigencias y a las nuevas violaciones del derecho internacional a nivel

\footnotetext{
${ }^{9}$ Traducción: Teniendo en cuenta que la expression the law of nations, el llamado derecho de las gentes, corresponde al derecho internacional consutudinario y debido a que los actores habian fundamentado la accion en juicio sobre la costumbre, para determinar su propia jurisdiccion el juez ha tenido que acertar la naturaleza consuetudinaria de la prohibicion de la tortura.
} 
legislativo, sino bien dejada a la exclusiva competencia jurisprudencial. De hecho, en los últimos años, las cortes federales han tenido que enfrentarse a numerosas cuestiones relativas a la naturaleza de dicha ley y a su compatibilidad con otras normas internas e internacionales que otorgan inmunidades, amnistías e indultos y a muchas otras cuestiones ligadas al ámbito de aplicabilidad de esta jurisdicción. El primer reto, como hemos visto en el caso Sosa, que las cortes federales han tenido que superar ha sido el relativo a los estándares o parámetros a la hora de determinar cuáles son los crímenes que pueden ser perseguidos en base a este acto.

Partiendo de este ultimo tema, tanto en la Jurisprudencia, como en la doctrina, podemos ver como han existido diferentes teorías. Desde 1980 han sido utilizados cinco estándares para determinar cuales crímenes pueden ser perseguidos a través del ATCA:

1. Customary International Law-Standard. En base a este criterio el ATCA puede ser activado en el caso de que un extranjero alegue haber sufrido un daño, siempre que este daño se concretice en una violación del derecho internacional o de los tratados en que los Estados Unidos son parte. Bajo este parámetro, cada violación del derecho internacional consuetudinario podría ser considerada suficiente para activar la tutela prevista por el ATCA.

2. El criterio del Universal and Obligatory Standard. Este criterio, mayormente utilizado por las cortes, reduce el ámbito de aplicación de la norma a las serias y graves violaciones de derecho internacional. Debemos reconocer que este criterio tiene la ventaja de adaptarse a la evolución del derecho internacional en cuanto permite que las nuevas violaciones, consideradas serias y graves del derecho internacional, que crean obligaciones universales para los Estados, entren en automático como crímenes punibles a través de la jurisdicción civil universal.

3. El criterio del Jus Cogens Standard. Este criterio permite, con algunas excepciones, incluir en el listado de los crímenes que puedan ser juzgados con el ATCA solo las normas que a nivel internacional llegan a ser normas de Jus Cogens, es decir, la parte del derecho internacional inderogable o modificable, de carácter superior. Obviamente, restringiendo demasiado el ámbito de aplicación del ATCA, no puede en principio ser considerado un criterio adecuado, teniendo en cuenta la lentitud con la que una norma de derecho internacional llega a ser considerada como norma de jus cogens. Esto no permitiría adaptarse con las graves y diferentes amenazas a la paz y la seguridad hoy en día existentes. Un ejemplo de lo anterior es el caso Sosa v. Alvarez Machain:

understood that the district courts would recognize private causes of action for certain torts in violation of the law of nations, . . . courts should require any claim based on the present-day law of nations to rest on a norm of international character accepted by the civilized world and defined with a specificity 
comparable to the features of the 18th-century paradigms we have recognized (Sosa v. Alvarez-Machain, 542 U.S. $692(2004)^{10}$.

4. Teoría aun más restrictiva es aquella propuesta por el Profesor Modeste Sweeney el cual afirmó que el ATCA debería activarse solo en caso de violaciones de la ley de las naciones entendida como los mismos parámetros utilizados cuando fue creada la ley. Siguiendo esta teoría, las cortes federales podrían empezar un juicio civil por los crímenes que en el momento de la creación del ATCA se configuraban como violaciones de un tratado o de la ley de las naciones o el derecho internacional conocido al estado de la redacción del ATCA.

5. Un último criterio es: The International Law Standard, que contrariamente a los dos anteriores, ampliaría demasiado el ámbito de aplicación de la norma sobre la jurisdicción civil. De hecho prevé que el ATCA se podría aplicar en todos los casos de violaciones de derechos individuales, reconocidos por el Derecho Internacional como tales y reconocidos a nivel nacional por los Estados.

Pasando al análisis sobre la aplicabilidad del ATCA en relación a los crímenes, tort committed in violation of the law of nations, las cortes federales han determinado la base jurídica sobre la activación del acto en los diferentes delitos. Partiendo del crimen de Genocidio en el caso Kadic vs. Karadzic de 1996, la corte federal de New York ha reconocido que: "The aftermath of the atrocities committed during the Second World War, the condemnation of genocide as contrary to international law quickly achieved broad acceptance by the community of the nations ${ }^{11}$ " ( Second Circuit; 1996: 241) y por lo tanto ha admitido que el crimen de genocidio es un delito sujeto de juicio bajo el ATCA, siempre que sea un delito cometido en larga escala.

Pasando a los crímenes en contra de la humanidad, en la misma sentencia de Kadic vs. Karadzic, la corte se ha expresado sobre estos delitos determinando que así como en el caso del genocidio, estos crímenes forman parte de derecho penal internacional formalmente reconocidos por parte de los Tribunales Penales Internacionales de la exYugoslavia, de Rwanda y por la Corte Penal Internacional y por lo tanto están incluido entre las violaciones previstas por el ATCA. Estos crímenes han sido objeto de muchos juicios delante de las cortes federales estadounidenses, entre todos se pueden mencionar los casos John Doe vs. Unocal y Tel- Oren vs. Libyan Arab Republic este último basado sobre el delito de Apartheid, un delito incluido en el listado de los crimenes en

\footnotetext{
${ }^{10}$ Traducción: entiendo que los tribunales de distrito reconocerían en juicios privados para ciertos actos ilícitos, en violación de la ley de las naciones,. . . los tribunales deberán aceptar cualquier reclamo basado en la ley actual de las naciones que sea una norma de carácter internacional aceptada por el mundo civilizado y definida con una especificidad comparable a las características de los paradigmas reconocidos en el siglo XVIII.

${ }^{11}$ Traduccion: Las consecuencias de las atrocidades cometidas durante la Segunda Guerra Mundial, la condena del genocidio como contraria al derecho internacional alcanzó rápidamente una amplia aceptación por la comunidad de las naciones
} 
contra de la humanidad.

Así como por el crimen de genocidio, la corte ha establecido que para la activación de la norma sobre la jurisdiccion civil universal, en los casos de crimen en contra de la humanidad, estos delitos deben ser cometidos en gran escala, siguiendo un plan directo al asesinato o exterminio en contra de una población o un grupo de personas.

En lo relativo a los crímenes de guerra, hay una diferencia fundamental con los primeros casos analizados al inicio de este párrafo. La peculiaridad respecto a los otros delitos radica en el hecho de que estos últimos necesitan un elemento para que se puedan configurar, es decir el conflicto armado o una guerra. Además se necesita una violación del derecho internacional humanitario, la ley de guerra, que establece las conductas prohibidas durante un conflicto interno o internacional, lo que se identifica través del llamado jus in bello. Actualmente el derecho internacional humanitario está dividido en dos pilares fundamentales: la ley de La Haya y las convenciones de Ginebra de 1949..

En el caso Kadic vs. Karadzic, pasando al análisis de los crímenes que se identifican como de guerra, la corte federal ha declarado que: "atrocities of the types alleged here have long been recognized in international law as violations of the law of war $^{12}$ " (Second Circuit; 1996:242). Por lo tanto, una vez establecido el hecho de que las convenciones de Ginebra habían sido reconocidas y ratificadas por 180 Estados, entre ellos Estados Unidos, la Corte, en consecuencia afirmó que los crímenes cometidos en violación de dichas normas pueden ser juzgados bajo el ATCA.

El crimen de tortura ha sido objeto de númerosos juicios por parte de las cortes federales partiendo del caso Filartiga vs. Peña-Irala. En este caso se había determinado que un acto de tortura cometido por parte de un oficial de un Estado en el momento de actuar una detención, viola las normas establecidas por el derecho internacional de los Derechos Humanos y por lo tanto determina una violación de la ley de las naciones y activa la tutela prevista del ATCA. Después del caso Filartiga, la protección contra los actos de tortura fue reforzada, por parte del congreso a través de la creación de una norma llamada Torture Victim Protection Act de 1991 que confirma la aplicabilidad del ATCA en los casos de tortura.

Esta norma ha clarificado no solamente los elementos constitutivos del crimen de tortura, con algunas diferencias respecto a la convenciones internacionales que prohíben la tortura, sino también las circunstancias a través de las cuales se pueden activar los juicios federales. En particular ha determinado que también un solo caso aislado de tortura, puede ser objeto de juicio delante de los tribunales federales. Como explica Koebele: «According with Cassese, opposed to genocide or crimes agaist humanity the prohibition agaist torture is not limited to atrocities on

\footnotetext{
${ }^{12}$ Traducción: Las atrocidades como las que estan alegadas aquí han sido reconocidas en el derecho internacional como violaciónes del derecho de guerra.
} 
large scale; istead every single instanse of torture amounts to a violation of international law ${ }^{13}$ » (Koebele; 2009:101).

Finalmente, algunos de los crímenes que pueden determinar un juicio delante de una corte federal sobre la base jurídica del ATCA se refieren a las violaciones cometidas en contra de los estándares internacionales establecidos por la protección de los trabajadores. La organización ILO, Internacional Labor Organization ha, de hecho establecido algunos estándares, determinados por el derecho internacional en tema de los derechos laborales. Estos principios y reglas han sido aceptadas por parte de la comunidad internacional, recogidas en convenciones internacionales y ratificadas por los Estados Unidos, como la Declaración de 1998: Declaration on Fundamental Principles and Rights at Work. En esta declaración se reconocen cuatros derechos fundamentales:

1. La eliminación del trabajo forzoso y obligatorio;

2. La eliminación de las discriminaciones;

3. La efectiva abolición del trabajo de niños;

4. La libertad de asociación y el reconocimiento de los derechos colectivos.

La violación de estos principios determina una violación del derecho internacional aceptado por los estados y puede tener como consecuencia la instauración de juicios delante de los tribunales estadounidenses como en el caso Unocal, que será analizado en los siguientes párrafos.

Desde mi punto de vista, el aspecto más rescatable del análisis de este acto es que la utilización actual del mismo se encuentra en camino hacia la creación de una responsabilidad de las empresas en la comisión o participación en un crimen internacional con el apoyo de un gobierno o de los aparatos militares. La cuestión relativa a la responsabilidad de las empresas ha sido tratada en diferentes ocasiones por las cortes federales empezando por el caso John Doe vs. Unocal que resulta también ser esencial para dar una visión del estado actual de la utilización del ATCA en tema de Derechos Humanos, y ha venido progresando en el periodo postSosa.

Partiendo del presupuesto de que esta ultima cuestión no pueda ser resuelta solamente con el auxilio del derecho internacional, las cortes federales se están moviendo en la dirección de evaluar la posibilidad de que pueda existir una responsabilidad para las empresas, partiendo del derecho nacional.

\footnotetext{
${ }^{13}$ Traducción: De acuerdo con Cassese, contrariamente al genocidio o a los crímenes en contra de la humanidad, la prohibicion de tortura no se limita a las atrocidades en gran escala; de hecho cada caso de tortura concretiza una violación del derecho internacional
} 


\section{El Caso John Doe v. Unocal}

Partiendo de la pregunta que se plantea Chimene Keitner cuando analiza el caso John Doe: "who benefits from a particular international law violation?» ¿Quién realmente $y$ en concreto se beneficia de las violaciones al derecho internacional?, podemos ver como:

ATS cases against corporate defendants can serve the function of redistributing the profits of abuses from those who committed or facilitated such abuses to their victims. Basic principles of unjust enrichment also animate this line of cases. Fifth, the Unocal line of cases also addresses a governance gap by articulating applicable standards of conduct and deterring corporations from engaging in unlawful conduct, including knowingly providing substantial assistance to human rights violators (which I have argued elsewhere is the applicable international law standard for aiding and abetting liability) ${ }^{14}$ (Keintner; 2012: 1016).

Es evidente que cuando se comete un crimen internacional, muchos son los actores que resultan ser involucrados en la comisión de dichos actos. Los casos llevados a cabo por los tribunales federales han evidenciado la importancia de redistribuir el enriquecimiento injusto de cada actor implicado y entre ellos las empresas.

Pasando al análisis del caso Unocal sobre los crímenes cometidos en Birmania en los años 90's por parte del gobierno militar, podemos ver como la sociedad Unocal se vio implicada en ellos de forma activa dando al gobierno militar un papel muy importante para la actuación de un proyecto relativo a la construcción y defensa de un gasoducto. El gobierno, en actuación de dicho proyecto y con el uso de la fuerza, obligó a la población a trabajar en la construcción de dicha instalación. Bajo su control, la situación degeneró y durante varios años se cometieron muchos crímenes en contra de la humanidad y de tortura para las personas que no querían prestar su colaboración. En 1996, algunas víctimas llevaron la cuestión frente al Tribunal del distrito de California llamando en causa a la sociedad californiana Unocal Corporation, que había propuesto al gobierno militar ejecutar el proyecto, también se llamó en causa a otras sociedades, como la nacional Myanmar Oil \& Gas Enterprise y a la trasnacional francesa Total S.A., que habían favorecido este plan en contra de la población civil.

En su decisión de primer grado, la Corte establecía que para entrar en el ámbito de actuación del ATCA y poder establecer la responsabilidad de una empresa en un crimen internacional cometido por miembros del gobierno o de la milicia, era necesario que la violación fuera realizada por

\footnotetext{
${ }^{14}$ Traducción: Los casos basados en ATS contra acusados corporativos pueden cumplir la función de redistribuir las ganancias de los abusos a los que cometieron o facilitaron estos abusos sobre las víctimas. Los principios básicos de enriquecimiento ilícito también pueden reflejase en estos casos. En quinto lugar, la línea de Unocal también evidencia un déficit de gobernanza mediante la articulación de las normas de conducta aplicables para disuadir a las empresas de incurrir en una conducta ilegal, incluyendo a sabiendas proporcionar asistencia sustancial a los violadores de derechos humanos (que he sostenido en otro lugar es la norma aplicable del derecho internacional para determinar la responsabilidad y la complicidad).
} 
parte de un Estado con la participación de otros sujetos, como empresas y que este requisito podía ser considerado conseguido cuando se aprobara la existencia de una joint action que pudiera involucrar partes privadas. En este sentido, el Tribunal toma en consideración la formula de joint action que sigue, afirmando que:

[u]nder the joint action approach, private actors can be state actors if they are 'willful participant[s] in joint action with the state or its agents' ${ }^{15}$. (John Doe I v. Unocal Corp., 963 F. Supp. at 890, que ha sido citada en Fonda v. Gray, 707 F.2d 435, 437 9th Cir. 1983).

En este caso específico, la Corte de primera instancia no encontró que se había obtenido la prueba de la existencia de una tal participación, de las sociedades en las acciones actuadas por parte del gobierno militar. Con apelación propuesta el 18 de septiembre del 2002, el Tribunal de segunda instancia denegó el modelo de joint action adoptado por el tribunal de primera instancia. La cámara de apelación tomó en consideración la active participation, afirmando que en los crímenes de trabajo forzado, homicidio o violaciones, no sirve que la conducta típica sea puesta en existencia por parte de un órgano de un Estado. Para llegar a este resultado la Corte utilizó la praxis dada por la jurisprudencia de los tribunales penales internacionales para la ex-Yugoslavia, en el caso de Anto Furundzija, International Criminal Tribunal for the ex Yugoslavia (ICTY), 10 de diciembre 1998, Prosecutor v. Furundzjia, IT-95-17/1, y del Tribunal para Rwanda, en el caso Alfred Musema' International Criminal Tribunal for Rwanda (ICTR), Prosecutor v. Musema, 27 de enero 2000, ICTR-96-13-T. En base a esta jurisprudencia la Corte afirmó que la sociedad Unocal que había concedido la actuación del proyecto sobre la construcción del acueducto al gobierno militar, sabía que este último actuaría adoptando un programa de trabajo forzado y utilizando cada medio útil para la represión de la población disidente y debido a ello había participado directamente en los hechos criminales actuados por el gobierno militar.

La sentencia de apelación que proclamaba la responsabilidad de la sociedad UNOCAL ha sido en un primer momento anulada, con ordenanza del 13 febrero del 2003, y después, por las negociaciones llevadas a cabo por las partes, se consiguió la firma de un acuerdo secreto y, sobre la base de este acuerdo el procedimiento se declaró extinto.

Siguiendo esta línea, en el caso Sarei v. Rio Tinto, caso abierto en el año 2000 en contra de Rio Tinto Plc, sobre las operaciones que la empresa había hecho en la isla de Bougainville, Papua Nueva Guinea, la corte ha admitido una Corporate liability under the Alien Tort Statute.

No obstante, contrariamente a esta sentencia se pueden mencionar casos en que las cortes federales negaron esta visión. En el caso Kiobel v. Royal

\footnotetext{
${ }^{15}$ Traducción: bajo el enfoque de una joint action, los actores privados pueden ser actores estatales si son "participantes voluntarios de una acción conjunta con el Estado o con sus agentes".
} 
Dutch Petroleum del año 2010, la corte de apelación del segundo circuito determinó en sus conclusiones que el derecho internacional consuetudinario no reconocía una responsabilidad de las empresas en la comisión de una violación de la law of nations. En su discurso el juez Carbranes declaraba que:

No corporation has ever been subject to any form of liability (whether civil, criminal, or otherwise) under the customary international law of human rights. Rather, sources of customary international law have, on several occasions, explicitly rejected the idea of corporate liability. Thus, corporate liability has not attained a discernable, much less universal, acceptance among nations of the world in their relations inter se, and it cannot not, as a result, form the basis of a suit under the ATS16.

Esta postura ha sido posteriormente modificada en las sucesivas y más actuales sentencias en que las cortes federales están regresando hacia la línea seguida en Unocal dejando de hecho abiertas la puertas para determinar los criterios y los elementos necesarios para fundamentar una responsabilidad de las empresas involucradas en crímenes internacionales.

\section{Conclusiones}

Uno de los avances más importantes en tema de protección de los Derechos Humanos y prevención/represión de las muchas violaciones cometidas en su contra ha sido la utilización de la jurisdicción universal tanto en tema civil como penal. La jurisdicción civil, prevista actualmente sólo por la legislación estadounidense, ha logrado abrir una esperanza, por lo menos en la óptica de restablecimiento económico del daño para las víctimas de un crimen internacional, en un Estado que no tiene ningún elemento de conexión con el mismo crimen. Desde 1980 las múltiples demandas que las cortes federales han recibido, han dado nueva vida a un acto de 1789 que desde el momento de su creación ha tenido una función noble, la de otorgar justicia. Desafortunadamente, no todas las reclamaciones han determinado la responsabilidad civil por la comisión de uno de dichos actos y muy pocas sentencias han logrado una ejecución final. De todas formas, y consciente de estos resultados, destaco que el conjunto de las demandas han puesto en evidencia el papel fundamental jugado por la jurisdicción civil universal que ha logrado abrir una alternativa a las víctimas y ha hecho posible al menos una reparación económica.

Aunque Los avances en este tema han sido seguramente muy

\footnotetext{
${ }^{16}$ Traducción: Ninguna empresa ha sido objeto de ningún tipo de responsabilidad (ya sea penal civil, o de otra manera) con el derecho consuetudinario internacional de los derechos humanos. Por el contrario, las fuentes del derecho internacional consuetudinario han, en varias ocasiones, rechazado explícitamente la idea de la responsabilidad corporativa. Por lo tanto, la responsabilidad corporativa no ha alcanzado un apreciable, mucho menos universal, la aceptación entre las naciones del mundo en sus relaciones inter se, y no puede, por lo tanto, constituir la base de una demanda bajo el ATS.
} 
importantes, aún queda mucho camino que recorrer. No podemos olvidarnos de las críticas que rodean este sistema de jurisdicción universal. Críticas que pueden ser claramente resumidas partiendo de las que se refieren al sistema en su totalidad. Antonio Cassese en su artículo sobre la justicia penal internacional, habla de este sistema de jurisdicción como de una respuesta para la represión de algunos crímenes internacionales y que por lo tanto se revela como una alternativa importante y útil, pero que al mismo tiempo tiene dos límites fundamentales que no se pueden dejar de tomar en consideración: En primer lugar tiene el límite de ser actuada por parte de un solo Estado, que además «no tiene una competencia universal de carácter penal» (Cassese 2007: 4).

En este sentido, la jurisdicción universal en materia civil no está relacionada propiamente con el deber de justicia penal relativo a la investigación y sanción de los crímenes internacionales ni con el ejercicio de la jurisdicción universal en materia penal, aunque sí se encuentra relacionada con el derecho de las víctimas a una adecuada reparación y con el derecho a la verdad (Del Toro Huerta, 2007:326).

A estos límites se puede agregar el hecho de que no siempre las decisiones o sentencias de condena para el reo, con el fin de dar una indemnización a las víctimas, encuentran seguimientos.

The US Courts judging under the Alien Tort Claim Act are civil courts only empowered to decide on monetary compensation to victims. Amounts in some cases are considerable, but may not by effectively implemented. Some victims have found such recourse, even if limited and symbolic, very gratifying. Under ATCA, victims are enabled to sue their tormentors, or families of victims can sue killers, but only the Prosecutor can initiate investigations and prosecutions in the international criminal tribunals ${ }^{17}$.

Pasando a las críticas más cercanas a la norma que prevé esta jurisdicción, es decir el Alien Tort Claims Act, podemos ver que la mayoría de ellas se sustentan en la incertidumbre a cerca de sus elementos y requisitos esenciales para la activación. Debido a esto, las cortes han tenido que adaptar el texto originario del ATCA incorporado en el Judiciary ACT de 1789, y no utilizado por dos siglos, a las nuevas exigencias en que se perpetuán las nuevas formas de amenazas a la seguridad humana y con esto a la paz, a través de sentencias a veces contradictorias.

Estos límites se reflejan y crean un sistema de justicia civil universal frágil; un sistema basado en una norma que al estado actual deja muchas

\footnotetext{
${ }^{17}$ Traducción: Los tribunales de los EE.UU. autorizados a juzgar bajo el Alien Tort Claim Act, son los únicos tribunales civiles facultados para decidir sobre la indemnización monetaria a las víctimas. La cantidades de las cuales en algunos casos resultan ser importantes, pero no pueden aplicarse de manera efectiva. Algunas víctimas han encontrado este recurso, aunque sea de forma limitada y simbólica, muy gratificante. Bajo el ATCA, las víctimas puedan denunciar a sus verdugos, o las familias de las víctimas pueden demandar a los asesinos, pero sólo el fiscal puede iniciar investigaciones y enjuiciamientos en los tribunales penales internacionales.
} 
cuestiones sin resolver. Como ejemplo podemos mencionar la delicada cuestión relativa a la determinación de la compatibilidad de este acto con otras leyes internas o internacionales que otorgan inmunidades, amnistías o indultos a miembros de gobierno, jefes o ex jefes de estados, etc. El caso reciente del ex presidente Mexicano Ernesto Zedillo es emblemático de esta situación y enfatiza la fragilidad de un sistema que carece de reglas claras y precisas y que sobre todo no logra alejarse de las cuestiones puramente políticas, otorgando tal vez inmunidades también cuando hayan sido cometidos crímenes internacionales.

De todas formas, el recorrido y los progresos hechos por las cortes federales en los últimos años, sobre todo en tema del reconocimiento de una responsabilidad de las empresas en la comisión o participación en un crimen itnernacional, otorgan esperanza a las víctimas de obtener justicia.

Las respuestas que faltan, los criterios incompletos y poco claros, ahora más que nunca se quedan en las manos de los tribunales nacionales estadounidenses, sobre los cuales descansa el mismo desafío de la comunidad internacional, determinar el equilibrio entre las fuerzas, los poderes y las normas internas e internacionales sobre todo cuando lo que está en juego es la protección de los Derechos Humanos y la lucha contra la impunidad.

\begin{abstract}
Por todo ello, los jueces nacionales tienen un importante rol que cumplir al momento de valorar y ponderar los diferentes principios fundamentales del derecho internacional y el juego de las inmunidades; el desarrollo que pueda darse en el ámbito nacional será determinante para la construcción de soluciones de lege ferenda que permitan el equilibrio entre el principio de inmunidad de jurisdicción estatal y el deber de reparación por violaciones graves a los derechos humanos, y, en ello, habrá que valorar la eficacia o no que ha tenido el principio de jurisdicción universal en materia civil (Del Toro Huerta; 2006).
\end{abstract}

\title{
VI. Bibliografía
}

Beccaria, Cesare (2003): Dei delitti e delle pene (1764), Feltrinelli, Milano.

BEIGBEDER, YVES (2005): International Justice against Impunity Progress and New Challenges, Martinus Nijhiff Publishers, Koninklijke Brill NV, Leiden.

Buxbaum, Richard M. y David D. Caron (2010): "The Alien Tort Statute: An Overview of the Current Issues», Berkeley Journal of International Law, 28:2, 511-518.

CASSESE, ANTONIO (2007): La giustizia penale internazionale, UTET, Torino.

Del Toro huerta, Mauricio IVÁn (2007): «La jurisdicción universal en materia civil y el deber de reparación por violaciones graves a los derechos humanos", Anuario Mexicano de Derecho Internacional, 7, 315349. 
Del Toro huerta, Mauricio IVÁn (2006): Transcripción de la ponencia presentada en el Congreso Internacional de Derecho Internacional de los Derechos Humanos realizado del 23 al 26 de mayo de 2006, en el Instituto de Investigaciones Jurídicas de la UNAM, disponible en: http://biblio.juridicas.unam.mx/estrev/derint/cont/7/art/art9.htm

KEITNER, CHIMENE I. (2012): Some functions of Alien Tort Statute Litigation, Georgetown Journal of International Law, 43, 105-107.

Koebele, Michael (2009): Corporate Responsibility under The Alien Tort Statute, Enforcement of International Law through US Torts Law, Martinus NIJHOFF Publishers, Leiden.

TANZI, ATTILA (2010): Introduzione al diritto internazionale contemporaneo, terza edizione, CEDAM, Padova.

TOMUSCHAT, CHRISTIAN (2009): en Juan Soroeta Liceras: "Los derechos Humanos frente a la impunidad", cursos de Derechos Humanos de Donostia-San Sebastian, Volumen X, ARGITALPEN ZERBITZUA, 11-21, Universad Pais Vasco, Bilbao. 